\title{
Prescribing a Statin to a Cirrhotic Patient to Reduce Hepatic Decompensation and Improve Survival: Impossible You Say?
}

\author{
James H. Lewis
}

Published online: 10 July 2014

(c) Springer Science+Business Media New York 2014

\section{Why, sometimes I've believed as many as six impos- sible things before breakfast-Lewis Carroll, Alice in Wonderland. .}

It was not all that long ago that strongly held clinical opinions to avoid prescribing 3-hydroxy-3-methyl-glutarylCoA reductase inhibitors (statins) to patients with any degree of liver disease were the norm. Over the past decade, however, data have consistently supported the safety of statins in patients with chronic liver disease (CLD), including cirrhosis, and have transformed the impossible into not only the possible, but the commonplace [1-4]. It may have taken more than 20 years, but even the Food and Drug Administration (FDA) has changed the prescribing information labels of statins, eliminating the requirement for liver-associated enzyme monitoring in patients with normal liver-associated enzymes (LAEs) [5]. And an enlightened medical community understands that the cardioprotective and other benefits of statins outweigh the potential risks of these agents, even in patients with underlying CLD [6].

In this issue of Digestive Diseases and Sciences, Kumar et al. [7] from Boston take these benefits of statins an important step further. By demonstrating a significant reduction in the risk of hepatic decompensation and mortality rates among cirrhotics who took a statin for at least 3 months, they have provided important outcomes data that add to a growing database supporting the safe and favorable use of statins in patients with advanced liver disease $[1-4,6,8,9]$. The authors retrospectively compared 81 patients with biopsy-proven

J. H. Lewis $(\square)$

Georgetown University Hospital, Room M2408, 3800 Reservoir

Road, NW, Washington, DC 20007, USA

e-mail: lewisjh@gunet.georgetown.edu cirrhosis [the majority of whom were Child-Pugh-Turcotte (CPT) class A or B] who took statins for at least 3 months and were followed for up to 10 years. The control group, composed of cirrhotics who did not take statins, was well matched in a 2:1 ratio. The investigators report that over this time period, the primary endpoint of overall mortality in the statin group was significantly lower (37\%) compared to $50.6 \%$ in the controls. Only two individuals $(2.4 \%)$ taking statins received a liver transplant compared to $18 \%$ in the control group. Similarly, the secondary endpoint of hepatic decompensation was also statistically lower in those on a statin (30\%) compared to the controls $(40.5 \%)$.

\section{Improved Outcomes in Cirrhotics on Statins}

A number of additional interesting observations were made from this retrospective cohort study that confirm other published reports. The cause of death was infection and malignancy in both groups, with no significant differences between the two cohorts, in whom hepatocellular carcinoma (HCC) was present at baseline in 11 and $12 \%$, respectively. Interestingly, while ascites was observed more often in the control group (31 vs $16 \%$ ), the effect on variceal bleeding was the opposite, with a higher rate reported in those taking statins (11 vs $2 \%$ ). Since just over one-third of the patients had varices at the time of enrollment, the failure to reduce bleeding from varices was an unexpected and unexplained finding, especially since statins can reduce portal pressure after just 1 month of use [10]. There were no differences between the two groups in terms of developing jaundice or hepatic encephalopathy. Just over half of the patients were diabetic, consistent with the most common etiologies of cirrhosis in the two cohorts being non-alcoholic fatty liver disease (NAFLD) or cryptogenic cirrhosis; the latter often related to NAFLD. 
Thus, the authors have described what is essentially a natural history study on the use of statins in a cirrhotic population, although at the end of 10 years, only $15 \%$ of the statin cohort and only $10 \%$ of the controls were still being followed.

There were no apparent differences noted in the two major outcomes based on the type, dose or duration of the several statins used. Nevertheless, the duration of statin use needed to provide the benefits of preventing hepatic decompensation and prolonging survival cannot be determined from the discussed study, as the investigators did not analyze these outcomes for statins taken for more than 3 months. They justified the 3-month time frame based on Abraldes et al. [10] who reported a significant reduction in the hepatic venous pressure gradient after just 1 month of daily simvastatin. Nevertheless, their study leaves open the question of whether long-term use (i.e., years) of a statin provides any ongoing, additional or cumulative benefits compared to relatively short-term use (i.e., months).

The reduction in mortality was attributed by the investigators directly to one or more effects of the statins, since, according to univariate and multivariate analyses, coronary artery disease, diabetes, the presence of HCC, fatty liver or MELD scores did not influence outcome. Nonetheless, one of the limitations of this retrospective analysis was that onethird of both groups did not have a cause of death listed, which could bias data interpretation. Also of interest was that the use of a beta blocker at baseline was associated with a seemingly paradoxically increased risk of hepatic decompensation. As the investigators acknowledge, however, they only recorded whether or not a beta blocker was being taken at baseline, and disappointingly do not provide any information about whether these agents were continued or discontinued during the study. As a result, the risk of decompensation and mortality associated with a beta blocker in concert with a statin cannot be determined. Perhaps some patients stopped taking a beta blocker during the course of the study. Such knowledge might also help explain why the risk of variceal hemorrhage was not reduced among the statin users, despite the expected reduction in portal pressure attributed to their use [10]. Yet another limitation of their analysis involved the inability of the investigators to provide information regarding the efficacy of statins on the baseline levels of total cholesterol or LDL, as these data were not collected. It can be inferred that they were likely reduced, since statins maintain their cholesterol-lowering efficacy in CLD, up through at least CPT class A cirrhotic patients [4].

\section{How to Monitor LAEs in Patients with Cirrhosis Taking a Statin?}

Although the hepatotoxicity observed in animals during statin development led to the requirement for liver enzyme monitoring in patients, numerous studies published in the past several years have emphasized the safety of statins in patients with underlying CLD $[1-4,6]$. Furthermore, statins prevent HCC among other malignancies [11-13]. Beneficial effects of statins in patients with chronic hepatitis $C$ suggest that these compounds have specific antiviral effects of as well [14]. While the FDA has eliminated the need for liver enzyme monitoring in patients who have normal baseline liver tests [5], the question remains how patients with underlying liver disease or increased liver tests at baseline should be monitored while taking statins. It should be remembered that not all increases in aminotransferases necessarily reflect hepatotoxicity among statin users. In patients in whom myalgias may be present, finding abnormal muscle enzymes can help establish the etiology of a high AST, which is preferentially increased initially compared to ALT in those with muscle injury [15].

Subjects with underlying NAFLD and chronic hepatitis $\mathrm{C}$ among other CLDs tolerated high-dose pravastatin well with a doubling of an increased baseline ALT or AST (with up to five times the upper limits of normal permitted at enrollment) observed in only about half the number of statin recipients compared to placebo-treated patients with hypercholesterolemia over a 36-week treatment period (7.5 vs $12 \%$ ) [4]. A number of these patients had cirrhosis, but all were CPT class A, as class B and class C were an exclusion. The current analysis by Kumar et al. [7] extends these observations of using a statin in patients with more advanced cirrhosis by including those with CPT class B and class $\mathrm{C}$ cirrhosis, although the number of individuals who was class B or C was only about $30 \%$ of the total, with nearly all being class B. Only one class $\mathrm{C}$ patient was in the cohort receiving statins. Therefore, while little can be determined about the safety and potential benefits of statins in patients with the most advanced cirrhosis, MELD scores correlated well with the CPT classification in the mortality analysis for class A and class B subjects.

\section{Biopsy-Proven Versus Clinically Diagnosed Cirrhosis}

Kumar et al. [7] chose to evaluate only patients with biopsy-proven cirrhosis, eschewing less invasive means of diagnosing advanced fibrosis. Yet, their results can likely be extrapolated to patients in clinical practice who have not undergone liver biopsy, as many noninvasive tests are available to aid in the diagnosis of cirrhosis, such as the FibroScan, are becoming more commonplace, replacing the need for liver biopsy in many clinical instances [16]. Given the increasing number of studies supporting the safe use of statins in patients with underlying liver disease [6], including those with well-compensated cirrhosis [4], the current findings seem applicable to any patient in whom 
cirrhosis is diagnosed clinically. The longer time to death for the cohort on statins of 10.8 years compared to 6.3 years in the control group suggests that these agents confer a substantial survival advantage. For the subgroup of CPT class A patients, the time to death was delayed even longer, being more than twice as long (14.4 years compared to 7 years) for the statin users as compared to nonusers [7].

\section{Are Statins the New Aspirin for the Twenty-First Century?}

Statins reduce all-cause mortality, especially due to cardiovascular disease, in both high-risk and low-risk individuals [17]. Indeed, akin to the present study [7], the cardiovascular benefits of lipid lowering with statins have actually been greater among patients with mild-to-moderately increased baseline levels of alanine aminotransferase (ALT) compared to individuals with normal ALT values [18]. Moreover, a recent review of the Third National Health and Nutrition Examination Survey (NHANES III) mortality files reported that statin use was also associated with a significantly lower rate of liver-related mortality compared to non-statin users [8], which Kumar et al. [7] were able to confirm. Besides, there was no association between statin use and mortality from liver disease [8], confirming the overall rarity of severe statin-related hepatotoxicity [19]. Similarly, although Kumar et al. [7] were unable to define the specific causes of infection leading to death among their cohort, the lower mortality rate they observed is also in keeping with an analysis of the US Veterans Health Administration database that suggests cirrhotic patients taking statins have a significantly reduced risk of developing serious infections [9].

While Kumar et al. [7] were not able to assess any effect of statins on the subsequent development of HCC among their two cohorts, several case-control studies suggest a beneficial effect of statins in reducing the incidence of $\mathrm{HCC}$ in patients at risk due to viral hepatitis and diabetes [11-13]. As mentioned above, controlled observations support a significant reduction in portal hypertension with statins [10], and antiviral effects of these medications have been increasingly reported $[3,14]$.

\section{Should Statins Be Routinely Prescribed to Patients with Cirrhosis?}

With the addition of studies such as that by Kumar et al. [7], perhaps we should be considering the empiric use of a statin in all cirrhotic patients, and not just those with hypercholesterolemia, as a means of prolonging survival, preventing decompensation and reducing the risk of developing HCC. Is such a scenario possible? While it would be easy to call for additional studies to confirm the findings of reduced mortality in cirrhotics receiving a statin before considering their routine use in this setting, the time and effort required to perform such long-term follow-up studies would be substantial. With the current literature supporting safe use in CLD $[1-4,6]$ as well as substantial clinical benefits $[6,8-14]$ with no serious adverse consequences, we already have sufficient evidence to recommend statins in our patients with cirrhosis at this time. As the saying goes, "The difficult we do immediately. The impossible takes a little longer." [20]. The study by Kumar and colleagues certainly helps to substantially shorten this time span.

Conflict of interest None.

\section{References}

1. Vuppalanchi R, Teal E, Chalasani N. Patients with elevated baseline liver enzymes do not have a higher frequency of hepatotoxicity from lovastatin than those with normal baseline liver enzymes. Am J Med Sci. 2005;329:62-65.

2. Argo CK, Loria P, Caldwell SH, Lonardo A. Statins in liver disease: a molehill, an iceberg, or neither? Hepatology. 2008;48:662-669.

3. Bader T. The myth of statin-induced hepatotoxicity. Am J Gastroenterol. 2010;105:978-980.

4. Lewis JH, Mortensen ME, Zweig S, et al. Efficacy and safety of high-dose pravastatin in hypercholesterolemic patients with wellcompensated chronic liver disease: results of a prospective, randomized, double-blind, placebo-controlled, multicenter trial. Hepatology. 2007;46:1453-1463.

5. FDA Drug Safety Communication: Important safety label changes to cholesterol-lowering statin drugs. www.fda.gov/Drugs/ DrugSafety/ucm293101.htm. Posted Feb 28, 2012. Accessed May $1,2014$.

6. Lewis JH. Clinical perspective: statins and the liver-harmful or helpful? Dig Dis Sci. 2012;57:1754-1763.

7. Kumar S, Grace ND, Qamar AA. Statin use in cirrhosis: a retrospective cohort study. Dig Dis Sci. (Epub ahead of print). doi:10.1007/s10620-014-3179-2.

8. Younoszai Z, Li Z, Stepanova M, et al. Statin use is not associated with liver related mortality. Ann Hepatol. 2013;13:84-90.

9. Motzkus-Feagans C, Pakyz AL, Ratliff SM, et al. Statin use and infections in veterans with cirrhosis. Aliment Pharmacol Ther. 2013;38:611-618.

10. Abraldes JG, Albillos A, Banares R, et al. Simvastatin lowers portal pressure in patients with cirrhosis and portal hypertension: a randomized controlled trial. Gastroenterology. 2009;136:1651-1658.

11. El-Serag HB, Johnson ML, Hachem C, et al. Statins are associated with a reduced risk of hepatocellular carcinoma in a large cohort of patients with diabetes. Gastroenterology. 2009;136:1601-1608.

12. Singh S, Singh PP, Singh AG, et al. Statins are associated with a reduced risk of hepatocellular cancer: a systematic review and meta-analysis. Gastroenterology. 2013;144:323-332.

13. Lai SW, Liao KF, Lai HC, et al. Statin use and risk of hepatocellular carcinoma. Eur J Epidemiol. 2013;28:485-492.

14. Ali N, Allam H, Bader T, et al. Fluvastatin interferes with hepatitis $\mathrm{C}$ virus replication via microtubule bunding and a 
doublecortin-like kinase-mediated mechanism. PLOS ONE. 2013;8:e80304.

15. Nathwani RA, Pais S, Reynolds TB, Kaplowitz N. Serum alanine aminotransferase in skeletal muscle diseases. Hepatology. 2005;41:380-382.

16. Bonder A, Afdhal N. Utilization of FibroScan in clinical practice. Curr Gastroenterol Rep. 2014;16:372.

17. Ford I, Murray H, Packard CJ, et al. Long-term follow-up of the West of Scotland Coronary Prevention Study. N Engl J Med. 2007;357:1477-1486.

18. Tikkanen MJ, Fayyad R, Faergeman O, et al. Effect of lipid lowering with atorvastatin on cardiovascular outcomes in coronary heart disease patients with mild-to-moderate baseline elevations in alanine aminotransferase levels. Int $J$ Cardiol. 2013;168:3846-3852.

19. Russo MW, Hoofnagle JH, Gu J, et al. The spectrum of statin hepatotoxicity: experience of the drug-induced liver injury network. Hepatology. 2014. doi:10.1002/hep.27157.

20. Motto of the US Army Corps of Engineers: "The difficult we do immediately; the impossible takes a little longer." Adapted from de Calonne, CA: si c'est possible, c'est fait; impossible? cela se fera, (if a thing is possible, consider it done; the impossible? that will be done (quoted in J. Michelet Histoire de la Révolution Française (1847) I. ii. 8). http://www.answers.com. 